\title{
Multiple infections and diversity of cytoplasmic incompatibility in a haplodiploid species
}

\author{
L Mouton, H Henri, M Boulétreau and F Vavre \\ Laboratoire de Biométrie et Biologie Evolutive, Université Claude Bernard, 43 Bd du 11 Novembre 1918, 69622 Villeurbanne Cedex, \\ France
}

Cytoplasmic incompatibility $(\mathrm{Cl})$ is a sperm-egg incompatibility commonly induced by the intracellular endosymbiont bacterium Wolbachia that, in diploid species, results in embryo mortality. In haplodiploid species, two types of $\mathrm{Cl}$ exist depending on whether the incompatible fertilized eggs develop into males (male development (MD)) or abort (female mortality (FM)). $\mathrm{Cl}$ allows multiple infections to be maintained in host populations, and thus allows interactions to occur between co-infecting strains. In Leptopilina heterotoma, three Wolbachia strains coexist naturally (wLhet1, $w L h e t 2, w L h e t 3)$. When these three strains are all present, they induce a $\mathrm{Cl}$ of FM type, whereas wLhet 1 alone expresses a $\mathrm{Cl}$ phenotype intermediate between $\mathrm{MD}$ and FM. Here, we compare $\mathrm{Cl}$ effects in crosses involving insect lines sharing the same nuclear background, but harboring different mixtures of strains. Mating experiments showed that: (i) wLhet2 and wLhet3 also induce an intermediate $\mathrm{Cl}$ when acting alone, and show a bidirectional incompatibility; (ii) there is no interaction between the co-infecting strains in Cl expression; (iii) the diversity of Wolbachia present within a male host influences the expression of $\mathrm{Cl}$ : an increase in the number of strains is correlated with a decrease in the proportion of the MD type, which is also correlated with an increase in bacterial density. All these data suggest that the $\mathrm{Cl}$ of FM type results from a stronger effect than the MD type, which conflicts with the conventional hypotheses used to explain $\mathrm{Cl}$ diversity in haplodiploids, and could provide some new information about $\mathrm{Cl}$ mechanisms in insects.

Heredity (2005) 94, 187-192. doi:10.1038/sj.hdy.6800596

Published online 24 November 2004

Keywords: symbiosis; multiple infection; Wolbachia; cytoplasmic incompatibility; Leptopilina heterotoma

\section{Introduction}

Many endosymbionts cause reproductive abnormalities in their hosts (Hurst, 1993). The most common is Wolbachia, a strictly intracellular bacterium present in a wide range of arthropods (Rousset et al, 1992; Werren et al, 1995; Breeuwer and Jacobs, 1996; Oh et al, 2000; Werren and Windsor, 2000), and in filarial nematodes (Bandi et al, 1998). This maternally inherited endosymbiont is able to manipulate host reproduction in a variety of ways, which promotes its spread within host populations (Werren, 1997; Stouthamer et al, 1999; Stevens et al, 2001).

Cytoplasmic incompatibility $(\mathrm{CI})$ is the most common effect of Wolbachia: it has been described in crustaceans (Moret et al, 2001), arachnids (Breeuwer, 1997), and in a number of insects including mosquitoes, planthoppers, moths, beetles, wasps, and Drosophila (for a review, see Stouthamer et al, 1999). CI is a sperm-egg incompatibility, which occurs in crosses between males and females with differing Wolbachia infection status. It can be either unidirectional when infected males mate with uninfected females, or bidirectional in crosses where both males and females are infected with different CI-

Correspondence: L Mouton, Laboratoire de Biométrie et Biologie Evolutive (UMR-CNRS 5558), Université Claude Bernard - Lyon 1, 43 Bd du 11 Novembre 1918, 69622 Villeurbanne Cedex, France.

E-mail:mouton@biomserv.univ-lyon1.fr

Received 14 May 2004; accepted 31 August 2004; published online 24 November 2004 inducing Wolbachia strains (Hoffmann and Turelli, 1997). Although the molecular mechanism of CI has not yet been identified, a modification/rescue model has been proposed, which assumes the existence of two distinct bacterial functions (Werren, 1997): the modification (mod) function expressed in the male germ line during spermatogenesis, and the rescue (resc) function, expressed in the egg. At karyogamy, the modified paternal chromosomes are improperly condensed, and lost during early embryo development, unless the egg expresses the resc function corresponding to the Wolbachia strain present in the male (Breeuwer and Werren, 1993).

In diploid species, CI causes karyogamy failure, resulting in arrested development at an early embryonic stage (Callaini et al, 1997; Tram and Sullivan, 2002). In haplodiploid species, only fertilized eggs, which normally develop into diploid females, are subject to CI. When incompatible, they may encounter two types of CI: either they develop into males, like unfertilized eggs (male development (MD) type), or they die (female mortality (FM) type). In the MD type, the paternal set of chromosomes is totally eliminated, leading to haploid eggs that go on to develop into males (Breeuwer and Werren, 1990). The FM type is probably due to partial chromosome fragmentation, resulting in lethal aneuploidy (Breeuwer, 1997; Vavre et al, 2001).

One consequence of $\mathrm{CI}$ is to confer an advantage on the females containing the highest diversity of Wolbachia strains, because they can mate successfully with males of any kind (Frank, 1998). This advantage explains why 
multiple infections are so widespread in nature. Multiple infections provide an opportunity to investigate the phenotypic expression of different bacterial strains within the same host insect, their reciprocal incompatibility relationships, and their possible interactions. Such studies require insect lines with differing Wolbachia infection status, and have only been performed in a few species: Drosophila simulans (Merçot et al, 1995; Sinkins et al, 1995), Aedes albopictus (Dobson et al, 2001), Nasonia vitripennis (Perrot-Minnot et al, 1996; Bordenstein and Werren, 1998), and Callosobruchus chinensis (Kondo et al, 2002). These studies have concluded that the CI level increases with the number of strains present in the same male, with additive effects and almost no interaction (Perrot-Minnot et al, 1996; Bordenstein and Werren, 1998; Rousset et al, 1999; Dobson et al, 2001).

The situation may be more complex in haplodiploid species, since the type of CI may also vary as in NasoniaWolbachia associations, where the type of CI would depend on the host genetic background (Bordenstein et al, 2003). Bacterial factors may also be involved in CI type variation, as demonstrated in the Drosophila parasitoid Leptopilina heterotoma. This parasitic wasp harbors three different Wolbachia strains, designated wLhet1, wLhet2, and wLhet3 (Vavre et al, 1999). If all three strains are present together, they induce a complete CI of FM type, whereas whet1 alone induces an intermediate CI type between MD and FM, that is, some of the fertilized eggs die, whereas others become haploid (Vavre et al, 2000, 2001). Thus, the type of CI depends on the diversity of Wolbachia co-infecting the individual host, which makes interactions possible between the different strains.

In this paper, we further study the association between Wolbachia and L. heterotoma using two recently obtained doubly infected lines (wLhet1/wLhet 2 or wLhet $1 /$ whet 3 ) (Mouton et al, 2003), and one singly infected line (wLhet1) (Vavre et al, 2001). Three main questions addressed were as follows: (i) Do wLhet2 and wLhet3 also induce CI? (ii) What are the incompatibility relationships between strains? (iii) How does the diversity of Wolbachia strains present influence the expression of CI? Results are discussed with regard to data on bacterial density recently obtained in L. heterotoma (Mouton et al, 2003).

\section{Materials and methods}

\section{Insect strains and rearing}

The wasp L. heterotoma (Hymenoptera, Figitidae) develops as a solitary larval parasitoid of many Drosophila species. In the laboratory, parasitoids were reared on a Wolbachia-free strain of D. melanogaster originating from $S^{\text {te }}$ Foy-les-Lyon, France. Rearing and experiments were carried out at $20^{\circ} \mathrm{C}$, LD 12:12 and $70 \% \mathrm{RH}$.

We used a line of Leptopilina heterotoma originating from Antibes (France), which had become homozygous after 35 generations of sib mating. This A7(123) line harbors three different Wolbachia strains: wLhet1, wLhet2, and whet3 (Vavre et al, 1999). An uninfected A7(0), a singly infected A7(1) and two doubly infected sublines, A7(12) and A7(13), were derived from A7(123) a number of generations before the experiments (at least 10 generations), using antibiotic treatments by feeding parasitized Drosophila larvae a standard diet containing low concentrations of rifampicin (Vavre et al, 2001; Mouton et al, 2003). Since all these sublines derived from the same A7(123) inbred line, they shared the same nuclear background and differed solely with regard to their infection status.

\section{Male reproductive capacity}

Despite the fact that all the sublines were derived from the highly inbred A7 line, we performed a preliminary experiment to confirm their reciprocal nuclear compatibility and the uniformity of the male reproductive capacity. Isolated virgin A7(123) females (3-4 days old) were individually mated with males (5 days old) of each infection status $(\mathrm{A} 7(0), \mathrm{A} 7(1), \mathrm{A} 7(12), \mathrm{A} 7(13)$ and A7(123)) to avoid CI (15 couples per cross type). Mating was visually checked, and each female was then provided with a batch of 100 Drosophila larvae for $48 \mathrm{~h}$. After development, the number of adult Drosophila and male and female wasps emerging from them were counted, and the offspring sex ratio (SR) calculated individually ( $\mathrm{SR}=\%$ males among the emerging wasps). Five control Drosophila groups were kept without wasps to estimate the natural egg-to-adult mortality among unparasitized Drosophila.

\section{Test for $\mathrm{Cl}$}

CI was tested in crosses between males (5 days old) and females (4 days old) of various infection statuses. For each combination, 15 pairs of males and virgin females were isolated. After mating had been observed, each female was provided for $48 \mathrm{~h}$ with the Drosophila larvae emerging from 100 eggs in vials containing sufficient diet for further development (these conditions prevent multiple parasitic infestation of the Drosophila larvae). A total of 10 control host groups were kept unparasitized to estimate the natural mortality of Drosophila in the absence of parasitoids. After development, the numbers of Drosophila, and of male and female wasps emerging from each vial, were recorded (a few crosses where fewer than five wasps had emerged were not taken into consideration in the data analysis). Comparison with compatible crosses involving males and females with the same infection status allowed us to estimate both the CI level and the percentage of MD CI type.

$$
\text { CIlevel }=\left(1-\frac{F}{F_{\mathrm{C}}}\right) \times 100 \text {. }
$$

The percentage of the MD type is the percentage of fertilized eggs that have been haploidized by Wolbachia. It takes into account the excess number of males produced by incompatible crosses $\left(M-M_{C}\right)$, and the number of fertilized eggs that suffered $C I\left(F_{C}-F\right)$.

$$
\text { Percentage of MD type }=\frac{M-M_{\mathrm{C}}}{F_{\mathrm{C}}-F} \times 100 .
$$

where $F_{C}$ is the mean number of females emerging from compatible crosses; $M_{C}$ the mean number of males emerging from compatible crosses; $F$ the number of females emerging from the incompatible cross tested; and $M$ the number of males emerging from the incompatible cross tested. 


\section{Results}

\section{Male reproductive capacity}

There were no statistically significant differences between the numbers of Drosophila, or of male and female wasps that emerged from any of the crosses involving A7(123) females and males of various infection statuses (Table 1). This demonstrated that there was no nuclear incompatibility between the different L. heterotoma lines, and that the reproductive capacity of the males was the same regardless of their infection status.

\section{Tests for $\mathrm{Cl}$}

We compared the phenotypic effects of wLhet 2 and wLhet3, and tested the influence of the diversity of Wolbachia strains on CI expression. Table 2 shows the number of male wasps, the total offspring, the SR, the level of CI, and the percentage of the MD CI type, and Figure 1 shows the number of Drosophila, and of male and female wasps emerging from each cross. Cross number 1 is the control cross between A7(0) males and females. In all other crosses, the SR was highly malebiased, with few or no females emerging, indicating a very high CI level. Moreover, the numbers of Drosophila

Table 1 Test for the reproductive capacity of males with various infection statuses

\begin{tabular}{lrccc}
\hline Male (n) & $\begin{array}{c}\text { Number of } \\
\text { Drosophila }\end{array}$ & $\begin{array}{c}\text { Number of } \\
\text { males }\end{array}$ & $\begin{array}{c}\text { Number of } \\
\text { females }\end{array}$ & $S R$ \\
\hline A7(0) (12) & $9.58 \pm 1.85$ & $18.17 \pm 1.25$ & $58.92 \pm 2.90$ & $0.310 \pm 0.069$ \\
A7(1) (15) & $11.93 \pm 2.49$ & $19.73 \pm 1.80$ & $54.53 \pm 2.33$ & $0.363 \pm 0.134$ \\
A7(12)(15) & $15.67 \pm 3.19$ & $16.00 \pm 1.43$ & $51.73 \pm 2.03$ & $0.307 \pm 0.089$ \\
A7(13)(11) & $16.00 \pm 3.44$ & $15.82 \pm 1.38$ & $51.64 \pm 2.86$ & $0.305 \pm 0.058$ \\
A7(123) (10) & $15.00 \pm 3.40$ & $17.30 \pm 1.80$ & $49.40 \pm 3.09$ & $0.342 \pm 0.065$ \\
& & & & \\
ANOVA & $\mathrm{F}_{4,58}=0.88$ & $\mathrm{~F}_{4,58}=1.14$ & $\mathrm{~F}_{4,58}=2.01$ & $\mathrm{~F}_{4,58}=1.14$ \\
& $P=0.4841$ & $P=0.3472$ & $P=0.1050$ & $P=0.3484$
\end{tabular}

Numbers of Drosophila and of male and female wasps emerging, and SR (percentage of male) in crosses between A7(123) females and $\mathrm{A} 7(0), \mathrm{A} 7(1), \mathrm{A} 7(12), \mathrm{A} 7(13)$ or $\mathrm{A} 7(123)$ males (mean \pm standard error). $n$ indicates the number of couples tested. One-way ANOVA was carried out after arcsine square root transformation for SR. were far higher and wasp production lower, which is typical for a CI phenotype of FM type. In some crosses, the increase in the production of males indicates that some incompatible eggs developed into males.

Effects of wLhet2 and wLhet3: Crosses 2 and 4 were used to find out whether wLhet3 and wLhet 2 are able to induce CI. However, since we failed to obtain lines singly infected with these strains, their effects were in fact tested in the presence of wLhet1. A male-biased offspring SR and high reduction in the production of females indicate that both strains induced CI. Moreover, far fewer offspring were produced than from the control cross, and the number of males was higher, indicating that the CIs induced by whet 2 and whet 3 when present separately were intermediate between MD and FM types, with approximately $30 \%$ of the fertilized eggs developing into males.

Crosses 3 and 5 also tested the CI induced by whet 2 and wLhet3, but in the presence of wLhet1, and of wLhet3

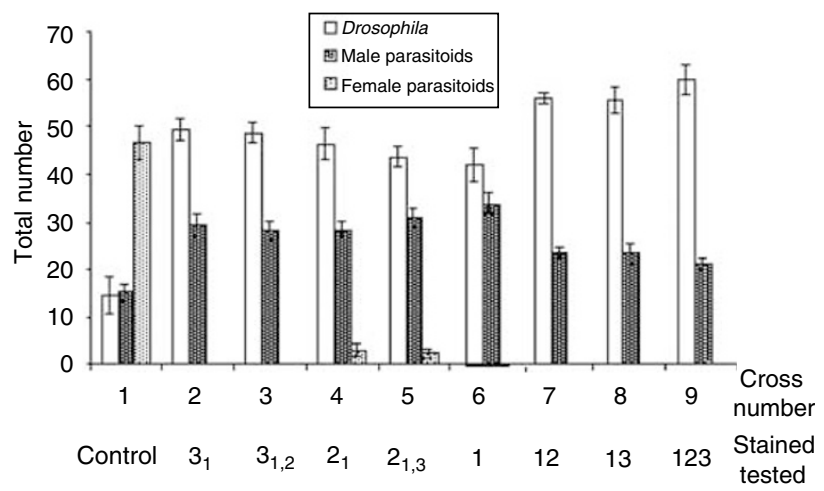

Figure 1 Numbers of Drosophila, and of male and female wasps emerging from crosses between males and females with various infection statuses (mean and standard error). Each cross type is identified by the corresponding cross number (see Table 2). The Wolbachia strain phenotypic effect tested is indicated for each cross: the bold number corresponds to the Wolbachia strain(s) (1 for wLhet1, 2 for wLhet2, and 3 for wLhet 3 ) and the superscript corresponds to the other Wolbachia strains also present in the male (for example, $3_{1}$ corresponds to the effect of whet3 in the presence of wLhet1).

Table 2 Tests for CI

\begin{tabular}{|c|c|c|c|c|c|c|}
\hline Cross number (n) & Cross $($ female $\times$ male $)$ & Number of male wasps & Total offspring & Sex ratio & CI level & $\% M D$ \\
\hline $1(8)$ & $\mathrm{A} 7(0) \times \mathrm{A} 7(0)$ & $15.1 \pm 1.7(\mathrm{a})$ & $61.7 \pm 3.8(\mathrm{a})$ & $0.246 \pm 0.029$ (a) & 0 & - \\
\hline $2(15)$ & $\mathrm{A} 7(1) \times \mathrm{A} 7(13)$ & $29.5 \pm 2.3(b, c)$ & $29.6 \pm 2.3(b)$ & $0.998 \pm 0.002(b)$ & $99.8 \pm 0.1$ (a) & $31.0 \pm 4.9(\mathrm{a}, \mathrm{b})$ \\
\hline $3(14)$ & $\mathrm{A} 7(12) \times \mathrm{A} 7(123)$ & $28.2 \pm 1.9(\mathrm{~b}, \mathrm{~d})$ & $28.3 \pm 1.9(\mathrm{~b}, \mathrm{~d})$ & $0.998 \pm 0.002(b)$ & $99.8 \pm 0.2$ (a) & $28.2 \pm 4.0(\mathrm{~b}, \mathrm{c})$ \\
\hline $4(15)$ & $\mathrm{A} 7(1) \times \mathrm{A} 7(12)$ & $28.5 \pm 1.6(b)$ & $31.5 \pm 2.3(b)$ & $0.920 \pm 0.027(\mathrm{c})$ & $93.8 \pm 2.7(b)$ & $31.9 \pm 4.7(\mathrm{a}, \mathrm{c})$ \\
\hline $5(12)$ & $\mathrm{A} 7(13) \times \mathrm{A} 7(123)$ & $31.1 \pm 2.1(\mathrm{~b}, \mathrm{c})$ & $33.5 \pm 2.4(\mathrm{~b})$ & $0.937 \pm 0.024$ (c) & $94.8 \pm 2.0(\mathrm{~b})$ & $36.6 \pm 4.9(\mathrm{a}, \mathrm{c})$ \\
\hline $6(12)$ & $\mathrm{A} 7(0) \times \mathrm{A} 7(1)$ & $34.1 \pm 2.1$ (c) & $34.2 \pm 2.2(\mathrm{~b})$ & $0.998 \pm 0.002(b)$ & $99.8 \pm 0.2$ (a) & $40.9 \pm 4.6$ (a) \\
\hline $7(14)$ & $\mathrm{A} 7(0) \times \mathrm{A} 7(12)$ & $23.4 \pm 1.1(\mathrm{~d}, \mathrm{e})$ & $23.4 \pm 1.1(\mathrm{c}, \mathrm{d})$ & $1 \pm 0(b)$ & $100.0 \pm 0.0(\mathrm{a})$ & $17.9 \pm 2.5(\mathrm{~b}, \mathrm{~d})$ \\
\hline $8(13)$ & $\mathrm{A} 7(0) \times \mathrm{A} 7(13)$ & $23.3 \pm 2.1(\mathrm{~d}, \mathrm{e})$ & $23.3 \pm 2.1(\mathrm{c}, \mathrm{d})$ & $1 \pm 0(b)$ & $100.0 \pm 0.0(\mathrm{a})$ & $18.8 \pm 4.1(\mathrm{~b}, \mathrm{~d})$ \\
\hline \multirow[t]{2}{*}{$9(12)$} & $\mathrm{A} 7(0) \times \mathrm{A} 7(123)$ & $21.1 \pm 1.3(\mathrm{a}, \mathrm{e})$ & $21.2 \pm 1.3(\mathrm{c})$ & $0.996 \pm 0.004(b)$ & $99.8 \pm 0.2(\mathrm{a})$ & $12.9 \pm 2.7(\mathrm{~d})$ \\
\hline & Statistical test & $\begin{array}{c}\mathrm{F}_{8,106}=7.74 \\
P<0.0001\end{array}$ & $\begin{array}{c}\mathrm{F}_{8,106}=22.13 \\
P<0.0001\end{array}$ & $\begin{array}{c}H_{8}=71.47 \\
P<0.0001\end{array}$ & $\begin{array}{c}H_{7}=46.02 \\
P<0.0001\end{array}$ & $\begin{array}{c}H_{7}=31.12 \\
P<0.0001\end{array}$ \\
\hline
\end{tabular}

Results of crosses between males and females with various infection statuses. Mean \pm standard error of numbers of male wasps, total offspring production, SR (percentage of males), CI level, and percentage of MD type. $n$ indicates the number of couples tested. Parametric tests were carried out for the number of male wasps and total offspring (one-way ANOVA and least significant difference tests), and nonparametric tests for the three other parameters (Kruskal-Wallis and Mann-Whitney tests). Means marked with the same letter are not significantly different $(P=0.05)$. 
or wLhet2, respectively. Similar results were obtained in crosses 2 and 3 for the effect of wLhet3, and in crosses 4 and 5 for the effect of whet 2 , indicating that, when coinfecting males, both these strains exert their mod (germline modification) effect on sperm independently. The $\mathrm{CI}$ in crosses 3 and 5 suggests that the rescue functions of wLhet 2 and wLhet 3 are distinct, as neither was able to rescue the sperm modified by the other. This was confirmed by the bidirectional incompatibility in reciprocal crosses between the $A 7(12)$ and $A 7(13)$ lines (Table 3). These crosses also confirm that the CIs induced by whet 2 and wLhet 3 are intermediate between the MD and FM types, since the male production was significantly higher in incompatible crosses, even though the percentage of the MD type was lower than previously observed.

Influence of Wolbachia diversity on $\mathrm{Cl}$ : We studied the influence of the diversity of the bacteria on the effect induced in the host by crossing uninfected females with males harboring $0,1,2$ or 3 Wolbachia strains.

When alone, wLhet1 (cross 6) induced a CI intermediate between the FM and MD types, and an increase in the number of male wasps. We estimate that $40.9 \%$ of the fertilized eggs became haploid, and that $59.1 \%$ died, which was consistent with a previous observation (Vavre et al, 2001).

In crosses involving doubly infected males (crosses 7 and 8), no females emerged, and the number of emerging males was intermediate between crosses 1 and 6 . Therefore, when mated with uninfected females, A7(12) and A7(13) males induced a complete CI that was intermediate between the FM and MD types. The percentage of the MD CI type was the same in both these crosses, but was less than $20 \%$ of the total CI, which was lower than in cross 6.

When the males were triply infected (cross 9), no females emerged and the number of male wasps was intermediate between those in the control cross and in crosses 7 and 8, although none of the differences was significant. This suggests that even when triply infected males are used, a very few incompatible eggs can develop into males, a result that was not found in previous studies (Vavre et al, 2000, 2001). Altogether, these findings indicate that the percentage of males decreases as the diversity of Wolbachia strains increases.

\section{Discussion}

Specific effects of the three strains (crosses 2-6)

Our results demonstrate that all the three Wolbachia strains that infect $L$. heterotoma induce CI. Similar coexistence of CI-inducing strains has been reported in other natural multiple Wolbachia associations (Merçot et al, 1995; Perrot-Minnot et al, 1996; Bordenstein and Werren, 1998; Dobson et al, 2001; Kondo et al, 2002), and is consistent with theoretical models. The CI effect induced by each strain confers a fitness gain on the multiply infected cytotypes, because multiply infected eggs are compatible with sperm of any infection status, allowing multiple infections to spread and be maintained (Frank, 1998).

Looking in greater detail, it turns out that, when alone, all three strains induce a CI phenotype intermediate between the FM and MD types. Unfortunately, the ability of wLhet 2 and wLhet3 to induce CI could not be tested in singly infected lines, and the present results must therefore be interpreted with caution. Nevertheless, their specific CI phenotypes were expressed in the same way in doubly and triply infected males, suggesting that the strains, at least wLhet 2 and whet3, do not interact with regard to their resc or mod functions, and that the $\mathrm{CI}$ level induced by each strain does not depend on the presence of the other. This can be related to recent data about Wolbachia density in L. heterotoma, which show that the density of each strain is specifically regulated, and does not depend on the presence of other strains (Mouton et al, 2003). The independence of the density of each strain could account for the stability of CI level induced, regardless of the presence of co-infecting strains.

\section{Effects of multiple infections (crosses 6-9)}

When acting alone, each of the three strains induces a CI that is intermediate between the MD and FM types; when associated with wLhet 2 or wLhet3, wLhet1 induces a FM CI with a lower MD component; and when males are triply infected, nearly all incompatible eggs die, indicating an almost complete FM type (Vavre et al, 2000; present results). Thus, in L. heterotoma, an increase in the number of Wolbachia strains co-infecting a male results in a lower proportion of the MD type. At the population level, such increase in mortality may facilitate the spread

Table 3 Test for bidirectional incompatibility between whet 2 and wLhet3

\begin{tabular}{|c|c|c|c|c|c|c|}
\hline Cross $($ female $\times$ male $)(\mathrm{n})$ & Number of Drosophila & Number of male wasps & Total offspring & Sex ratio & CI level & $\% M D$ \\
\hline $\mathrm{A} 7(12) \times \mathrm{A} 7(12)$ & $33.0 \pm 3.1$ (a) & $12.3 \pm 0.9$ (a) & $42.4 \pm 3.3$ (a) & $0.298 \pm 0.025$ (a) & - & - \\
\hline $\mathrm{A} 7(13) \times \mathrm{A} 7(13)(10)$ & $30.4 \pm 3.6$ (a) & $12.3 \pm 1.3$ (a) & $47.5 \pm 3.9$ (a) & $0.260 \pm 0.022$ (a) & - & - \\
\hline $\mathrm{A} 7(12) \times \mathrm{A} 7(13)(12)$ & $57.5 \pm 2.4(\mathrm{~b})$ & $18.2 \pm 1.6(\mathrm{~b})$ & $18.2 \pm 1.6(\mathrm{~b})$ & $1.000 \pm 0.000(\mathrm{~b})$ & $100.0 \pm 0.0$ & $20.4 \pm 4.9$ \\
\hline $\mathrm{A} 7(13) \times \mathrm{A} 7(12)(12)$ & $61.4 \pm 1.9(\mathrm{~b})$ & $19.2 \pm 1.7(\mathrm{~b})$ & $19.3 \pm 1.6(\mathrm{~b})$ & $0.989 \pm 0.010(b)$ & $99.5 \pm 0.5$ & $20.3 \pm 4.4$ \\
\hline Statistical test & $\begin{array}{c}\mathrm{F}_{3,39}=34.80 \\
P<0.0001\end{array}$ & $\begin{array}{c}\mathrm{F}_{3,39}=6.146 \\
P=0.0016\end{array}$ & $\begin{array}{c}\mathrm{F}_{3,39}=34.30 \\
P<0.0001\end{array}$ & $\begin{array}{l}H_{3}=31.43 \\
P<0.0001\end{array}$ & $\begin{array}{c}U=78.00 \\
P=0.3178\end{array}$ & $\begin{array}{l}U=72.00 \\
P<0.9999\end{array}$ \\
\hline
\end{tabular}

Results of reciprocal crosses between A7(12) and A7(13) males and females. Mean \pm standard error of the numbers of Drosophila and of male wasps, total offspring production, SR (percentage of males), CI level, and percentage of MD type. $n$ indicates the number of couples tested. Parametric tests were carried out for the numbers of Drosophila, of male wasps, and of the total number of offspring (one-way ANOVA and least significant difference test), and nonparametric tests were used for the three other parameters (Kruskal-Wallis and Mann-Whitney tests). Means marked with the same letter are not significantly different $(P=0.05)$. 
of multiple infections, since theoretical models have demonstrated that the FM type makes Wolbachia more invasive than the MD type (Vavre et al, 2000).

This result also indicates that a single host genotype can express different CI types. Conversely, Bordenstein et al (2003) have demonstrated in two Nasonia species that the host genetic background may also account for variations in the CI type. Therefore, the expression of CI in haplodiploid species is probably under the control of complex nucleo-cytoplasmic interactions.

In the system we are studying here, an increase in Wolbachia diversity also leads to an increase in Wolbachia density (Mouton et al, 2003), making it impossible to distinguish between their relative influences on the CI type. However, previous studies have shown that in multiply infected males the CI level results from the addition of the individual CI effects of each strain (Perrot-Minnot et al, 1996; Bordenstein and Werren, 1998; Rousset et al, 1999; Dobson et al, 2001), leading to an increase of the total Wolbachia effect in multiple infections. Likewise, since the proportion of the FM CI type increases with the diversity of Wolbachia, the FM type is probably 'harsher' than the MD type. This contrasts with the hypothesis of Breeuwer (1997) and Vavre et al (2000), who proposed that the MD type could result from greater modification and the complete loss of paternal chromosomes, so that the fertilized eggs revert back to haploidy and develop as males, whereas the FM type would result from the milder modification and an incomplete loss of paternal chromosomes, resulting in the lethal aneuploidy of incompatible eggs. The results reported here actually suggest the contrary: the FM CI type tends to be associated with the harsher Wolbachia effect. This could result either from such an extreme modification of the paternal chromosomes that further embryo development fails for some reason, or from the effects of Wolbachia on the cytoskeleton and/or microtubule apparatus of the sperm, which are known to be involved in early embryonic mitosis (Kose and Karr, 1995).

While cytological studies have shown that haploidization does occur in the MD CI type, there is as yet no cytological evidence demonstrating that egg aneuploidy is responsible for inducing FM CI. Clearly, cytogenetic analyses in haplodiploids are required before we can fully understand the diversity of CI they can display, but our results also suggest that analyzing the CI in haplodiploids could be helpful in elucidating the general mechanisms of CI.

\section{Acknowledgements}

This study was partly supported by the Centre National de la Recherche Scientifique (IFR 41-UMR5558).

\section{References}

Bandi C, Anderson TJC, Genchi C, Blaxter ML (1998). Phylogeny of Wolbachia in filarial nematodes. Proc $R$ Soc Lond B 265: 5407-5413.

Bordenstein SR, Uy JJ, Werren JH (2003). Host genotype determines cytoplasmic incompatibility type in the haplodiploid genus Nasonia. Genetics 164: 223-233.
Bordenstein SR, Werren JH (1998). Effects of A and B Wolbachia and host genotype on interspecies cytoplasmic incompatibility in Nasonia. Genetics 148: 1833-1844.

Breeuwer JAJ (1997). Wolbachia and cytoplasmic incompatibility in the spider mites Tetranychus urticae and T. turkestani. Heredity 79: 41-47.

Breeuwer JAJ, Jacobs G (1996). Wolbachia: intracellular manipulation of mite reproduction. Exp Appl Acar 20: 421-434.

Breeuwer JAJ, Werren JH (1990). Microorganisms associated with chromosome destruction and reproductive isolation between two insect species. Nature 346: 558-560.

Breeuwer JAJ, Werren JH (1993). Effect of genotype on cytoplasmic incompatibility between two species of Nasonia. Heredity 70: 428-436.

Callaini G, Dallai R, Riparbelli MG (1997). Wolbachia-induced delay of paternal chromatin condensation does not prevent maternal chromosomes from entering anaphase in incompatible crosses of Drosophila simulans. I Cell Sci 110: 271-280.

Dobson SL, Marsland EJ, Rattanadechakul W (2001). Wolbachiainduced cytoplasmic incompatibility in single- and superinfected Aedes albopictus (Diptera: Culicidae). J Med Entomol 38: 382-387.

Frank SA (1998). Dynamics of cytoplasmic incompatibility with multiple Wolbachia infections. J Theor Biol 192: 213-218.

Hoffmann AA, Turelli M (1997). Cytoplasmic incompatibility in insects. In: O'Neill SL, Hoffmann AA, Werren JH (eds) Influential Passengers. Inherited Microorganisms and Arthropod Reproduction. Oxford University Press: Oxford pp 42-80.

Hurst LD (1993). The incidences, mechanisms and evolution of cytoplasmic sex ratio distorters in animals. Biol Rev 68 : 121-193.

Kondo N, Ijichi N, Shimada M, Fukatsu T (2002). Prevailing triple infection with Wolbachia in Callosobruchus chinensis (Coleoptera: Bruchidae). Mol Ecol 11: 167-180.

Kose H, Karr TL (1995). Organization of Wolbachia pipientis in the Drosophila fertilized egg and embryo revealed by antiWolbachia monoclonal antibody. Mech Dev 51: 275-288.

Merçot H, Llorente B, Jacques M, Atlan A, Montchamp-Moreau C (1995). Variability within the Seychelles cytoplasmic incompatibility system in Drosophila simulans. Genetics 141: 1015-1023.

Moret Y, Juchault P, Rigaud T (2001). Wolbachia endosymbiont responsible for cytoplasmic incompatibility in a terrestrial crustacean: effects in natural and foreign hosts. Heredity $\mathbf{8 6}$ 325-332.

Mouton L, Henri H, Boulétreau M, Vavre F (2003). Strainspecific regulation of intracellular Wolbachia density in multiply infected insects. Mol Ecol 12: 3459-3465.

Oh HW, Kim MG, Shin SW, Bae KS, Ahn YJ, Park HY (2000). Ultrastructural and molecular identification of a Wolbachia endosymbiont in a spider, Nephila clavata. Insect Mol Biol 9: 539-543.

Perrot-Minnot M-J, Guo LR, Werren JH (1996). Single and double infections with Wolbachia in the parasitic wasp Nasonia vitripennis: effects on compatibility. Genetics 143: 961-972.

Rousset F, Bouchon D, Pintureau B, Juchault P, Solignac M (1992). Wolbachia endosymbionts responsible for various alterations of sexuality in arthropods. Proc $R$ Soc Lond $B$ 250: 91-98.

Rousset F, Braig HR, O'Neill SL (1999). A stable triple Wolbachia infection in Drosophila with nearly additive incompatibility effects. Heredity 82: 620-627.

Sinkins SP, Braig HR, O'Neill SL (1995). Wolbachia superinfections and the expression of cytoplasmic incompatibility. Proc R Soc Lond B 261: 325-330.

Stevens L, Giordano R, Fialho RF (2001). Male-killing, nematode infections, bacteriophage infection, and virulence of cytoplasmic bacteria in the genus Wolbachia. Annu Rev Ecol Syst 32: 519-545. 
Stouthamer R, Breeuwer JAJ, Hurst GDD (1999). Wolbachia pipientis: microbial manipulator of arthropod reproduction. Annu Rev Microbiol 53: 71-102.

Tram U, Sullivan W (2002). Role of delayed nuclear envelope breakdown and mitosis in Wolbachia-induced cytoplasmic incompatibility. Science 296: 1124-1126.

Vavre F, Dedeine F, Quillon M, Fouillet P, Fleury F, Boulétreau M (2001). Within-species diversity of Wolbachia-induced cytoplasmic incompatibilities in haplodiploid insects. Evolution 55: 1710-1714.

Vavre F, Fleury F, Lepetit D, Fouillet P, Boulétreau M (1999). Phylogenic evidence for horizontal transmission of Wolbachia in host-parasitoïd associations. Mol Biol Evol 16: 1711-1723.
Vavre F, Fleury F, Varaldi J, Fouillet P, Boulétreau M (2000). Evidence for female mortality in Wolbachia-mediated cytoplasmic incompatibility in haplodiploïd insects. Epidemiology and evolutionary consequences. Evolution 54: 191-200.

Werren JH (1997). Biology of Wolbachia. Annu Rev Entomol 42: 587-609.

Werren JH, Windsor DM (2000). Wolbachia infection frequencies in insects: evidence of a global equilibrium? Proc $R$ Soc Lond $B$ 267: 1277-1285.

Werren JH, Windsor D, Guo L (1995). Distribution of Wolbachia among neotropical arthropods. Proc R Soc Lond B 262: 197-204. 